\title{
有限次元線形モデルを用いる最適同定法
}

\author{
河注 植* 古田勝 久** \\ * 東京工業大学大学院 東京都目黒区大岡山 2-12-1 \\ ** 東京工業大学工学部 東京都目黒区大岡山 2-12-1 \\ (昭和 46 年 5 月 25 日 受付)
}

\section{An Optimal Identification Method Using a Finite Dimensional Linear Model}

\author{
Joo Shik $\mathrm{HA}^{*}$ and Katsuhisa Furuta** \\ (* Graduate School, Tokyo Institute of Technology, Meguro-ku, Tokyo \\ (** Faculty of Engineering, Tokyo Institute of Technology, Megro-ku, Tokyo)
}

(Received May 25, 1971)

The identification can be considered as a problem to find the optimal model in a set of models: chosen a priori. As a criterion function, (1) response error criterion function, (2) equation error criterion function have been usually used. It has been known that only the response error criterion is applicable when the information about the dynamical structure of the system to be identified is not available. But this criterion function is not much desirable in case when only limited number of observed data of input and output, contaminated by noises, are provided.

In the paper, the improved version of the response error criterion is proposed; i.e., the sum of the following three terms, (1) weighted square error of the initial state, (2) sum of the weighted square error between the input to the model and the observed input, (3) sum of the weighted square error between the output of the model and the observed output.

Minimization procedure with the proposed criterion function is achieved by two steps. First, determination of the optimal initial state and input to the model, which minimize the criterion function for a given model. This first step is known as the optimal tracking problem. Second, minimization of the criterion function with respect to the parameters of the model. This second step is a static optimization problem. The computational algorithm for a linear discrete system is presented.

The criterion function is also explained from the statistical viewpoint and found to be equivalent to the likelihood function in parameter estimation when the structure of the system is the same as that of the model.

The computational examples are given to illustrate the proposed identification method.

\section{1. 緒}

言

動的システムの同定は近年むすますとの重要性を增 し, 多くの研究がなされているが, 同定の定義は研究 者によって異なっている1). しかし，プラント（以後, 同定すべきシステムをプラントと呼ぶ）の入力, 出力 のよらなプラントの外部変数だけの情報を用いて同定 を行なら実験的同定法 (experimental identification)

* 現在, 韓国海洋大学勤務.
に限定して考光ると，同定の一般的定義としては，プ ラントに対してモデルとしてとりらるシステム（以後 モデルと呼ぶ）を要素とする集合（以後, 可能モデル 集合と呼ぶ）の中から，外部変数の測定結果を用いて， 適当湾与兄られた評価関数を最小炕する要素を求める ことであるといえる．また，同定に用いられる評価関 数としては, (1)実応答誤差のはん関数, (2)方程式誤差 のはん関数などが考兄られる。(2)の評価関数を用いる 場合には可能モデル集合がプラントと等価な要素 
(topologically equivalent model of the plant) を持 たなければならないのに対し，(1)の評価関数を用いる 場合はそのよらな必要はない. (2)の評洒関数を用いる 場合，モデル調整法を用いてもパラメータを同定する 安定な閉ループ系が作られることが知られているが2), 測定データが雑音に污されているときは一般に推定值 にバィアスを与える. Sacrison ${ }^{3)}$, Furuta ${ }^{4)}$ らはこれ に対する改良形評価関数を与えているが，この場合も プラントと等価なモデルが可能モデル集合に含まれる ことと長時間の測定データが必要である. (1)の評価関 数を用いる場合にも有限区間の測定データしか与兄ら れていないときは入力信号が正確に測定されなければ ならない.プラントと等価なモデルが可能モデル集合 に含まれることが知られていて，かつ，入出力の測定 誤差の統計的性質が既知の場合，同定問題は一般に統 計的パラメータ推定問題となる. この場合に用いられ る評価関数を特に統計的評価関数とも呼ぶ. 統計的評 価関数を用いて同定を行ならことは古くから提案され ている手法である. $\operatorname{Cox}^{5)}$ は most probablic estimate を用いて, 未知パラメータも状態変数と考光, 同 定問題を非線形推定問題として取扱ったが，これは非 線形 2 点境界值問題となるため，実際にはこの方法を 利用することが困難である。 Åström ${ }^{6)} ， K_{\text {ashap }}^{7)}$ は 最尤推定法を用いて，パルス伝達関数で記述されるプ ラントの入力に測定した入力信号以外に統計的雑音が 加わる場合，パルス伝達関数の未知パラメータを求め る方法を提案している. 同定と同時に制御も行なわれ る場合を考えると，一般に，同定と同時に過去の入力 と状態の推定も得られるのが望しい.しかし, Åström, Kashap はプラントを状態方程式で記述して拉らず, 単にパラメータ推定のみを目的としたため，過去の入 力と初期状態の推定を行なっていない. Mayne ${ }^{8)}$ Åström の結果を状態変数を用いて説明しているが過 去の入力と状態の推定は行なっていない.

本研究に特いては，プラントの入力信号之出力信号 の測定データのみが与えられた場合, 状態変数で記述 した次元離散值線形系を要素とする可能モデル集合の 中から最適モデルおよび過去の入力と状態（初期状態 を含さ）の推定を同時に求めるのに有効な評価関数を 提案し，この評価関数の下での同定アルゴリズムを示 す.すなわち，プラントの構造が末知で入出力の測定 誤差に関する統計的性質が未知の場合, 探索法を適用 するまえまでのパラメータ同定アルゴリズムを与える. ここで考光ている可能モデル集合は，プラントの次数 などは考虑せず，制御その他の条件からプラントに比 ベ低次のシステムを要素としているものとする.
ここで与えられる同定結果は適当な条件の下で最尤 推定になることが示される。この場合，本研究はこれ までの最尤推定による同定法を，初期状態と過去の入 力の推定も与えるように改良したものとみることもで きる。

\section{2. 同 定 問 題}

本報告では簡単のため単一入力, 単一出力離散值系 について述べるが，ここで提案する方法の多入力，多 出力系拉よび連続系への拡張は容易である. いま，プ ラントの入力 $v_{i}(i=0,1, \cdots, N-1)$ と出力 $z_{i}(i=0,1$, $\cdots N)$ が測定されたとする，このとき，本報告では， (1) 式のように記述される $n$ 次線形離散值系,

$$
\left.\begin{array}{l}
\boldsymbol{x}_{i+1}{ }^{m}=\boldsymbol{\Phi} \boldsymbol{x}_{i}{ }^{m}+\boldsymbol{g} u_{i} \\
y_{i}{ }^{m}=\boldsymbol{h}^{T} \boldsymbol{x}_{i}{ }^{m}
\end{array}\right\}
$$

ただし $, \boldsymbol{x}_{i}^{m}, \boldsymbol{g}, \boldsymbol{h}: n$-ベクトル, $\boldsymbol{\Phi}: n \times n$ 行列, $u_{i}^{m}$, $y_{i}^{m}:$ それぞれ入力と出力 (スカラー量).

を要素とする可能モデル集合の中から，(2) 式で表わ される評価関数を最小にする最適モデルを求める同定 問題を考える.

$$
\begin{aligned}
J= & \frac{1}{2}\left[\left\|\boldsymbol{x}_{0}{ }^{m}-\overline{\boldsymbol{x}}_{0}{ }^{m}\right\|^{2} w_{0}+\sum_{i=0}^{N-1}\left(v_{i}-u_{i}^{m}\right)^{2} w_{1 i}\right. \\
& \left.+\sum_{i=0}^{N}\left(z_{i}-\boldsymbol{h}^{T} \boldsymbol{x}_{i}{ }^{m}\right)^{2} w_{2 i}\right]
\end{aligned}
$$

ここで, $\overline{\boldsymbol{x}}_{0}{ }^{m}$ は a priori に考えられる $\boldsymbol{x}_{0}{ }^{m}$ の值で既 知だとする. $w_{0}, w_{1 i}, w_{2 i}$ は同定の目的または条件に よって適当に与えられる重み係数である (3. 参照). しかし，(1）式で記述されるモデルの中には同一入力 に対して同一出力を与える等価なモデルが数多く存在 するから，このような可能モデル集合の中から (3) 式 の評価関数を最小にする 1 つ最適モデルを決定する ことはできない，したがって，本報告では，(1) 式で 表わされるモデルの中で $\boldsymbol{\Phi}, \boldsymbol{g}, \boldsymbol{h}$ が (3). 式のように control canonical form ${ }^{9)}$ で記述され, かつ, $(\boldsymbol{\Phi}, \boldsymbol{h})$ が可観測であるモデルのみを要素とする集合を可能モ デル集合とするただし，プラシトと等価なモデルが 可能モデル集合に含まれることを前提にしない。

$$
\left.\boldsymbol{\Phi}=\left[\begin{array}{cc}
\vdots & \boldsymbol{I}_{n-1} \\
\mathbf{0} & \boldsymbol{I}_{n-1} \\
\cdots \ldots \ldots \ldots . . . & -\boldsymbol{k}^{T}
\end{array}\right], \begin{array}{l}
\boldsymbol{k}^{T}=\left[k_{1}, k_{2}, \cdots, k_{n}\right] \\
\boldsymbol{g}^{T}=[0,0, \cdots, 0,1] \\
\boldsymbol{h}^{T}=\left[h_{1}, h_{2}, \cdots, h_{n}\right]
\end{array}\right\}
$$

ここで, Tは転置， $\boldsymbol{I}_{n-1}$ は $n-1$ 次の単位行列を表わ す.この場合，最適モデルを決定する問題は (1)，(3) 式の拘束の下で (2) 式の評価関数を $\boldsymbol{h}, \boldsymbol{k}, \boldsymbol{x}_{0}{ }^{m}, u_{i}^{n}(i$ $=0,1, \cdots, N-1)$ について最小にする問題になる. こ れを次の 2 つの手順に分けて行なう。

(1) まず未知パラメータ $\boldsymbol{h}, \boldsymbol{k}$ が与えられたと仮定 
して（2）式を最小にする $\boldsymbol{x}_{i}^{m}, u_{i}{ }^{m}$ を求め, これを $\hat{\boldsymbol{x}}^{m}, \hat{u}_{i}$ とする (最適トラッキング問題).

(2) $\hat{\boldsymbol{x}}_{i}^{m}, \hat{u}_{i}$ を(2) 式に代入してこれを最小にする $\boldsymbol{h}, \boldsymbol{k}$ を求める (静的最適化問題).

このとき, (1) の解として $\hat{u}_{i}{ }^{m}, \hat{\boldsymbol{x}}_{i}{ }^{m}$ は (4)〜 (6) 式で 与えられる(10),11).

$$
\begin{aligned}
\hat{u}_{i}^{m}= & v_{i}-w_{1 i}{ }^{-1} \boldsymbol{g}^{T}\left(\boldsymbol{R}_{i+1} \hat{\boldsymbol{x}}_{i+1}-\boldsymbol{b}_{i+1}\right), \\
& i=0,1, \cdots, N-1 \\
\hat{\boldsymbol{x}}_{i+1}{ }^{m}= & \left(\boldsymbol{I}_{n}+w_{1 i}{ }^{-1} \boldsymbol{g} \boldsymbol{g}^{T} \boldsymbol{R}_{i+1}\right)^{-1}\left(\boldsymbol{\Phi} \hat{\boldsymbol{x}}_{i}{ }^{m}\right. \\
& \left.+w_{1 i}{ }^{-1} \boldsymbol{g} \boldsymbol{g}^{T} \boldsymbol{b}_{i+1}+\boldsymbol{g} v_{i}\right), i=0, \cdots, N-1 \\
\hat{\boldsymbol{x}}_{0}{ }^{m}= & M_{21}^{-1}\left(w_{10}{ }^{-1} \boldsymbol{g} \boldsymbol{g}^{T} \boldsymbol{b}_{1}+\boldsymbol{g} v_{0}\right) \\
& +\boldsymbol{M}_{22}^{-1}\left(\boldsymbol{\Phi}^{T} \boldsymbol{b}_{1}+\omega_{20} \boldsymbol{h} z_{0}+w_{0} \overline{\boldsymbol{x}}_{0}^{m}\right)
\end{aligned}
$$

ただし，

$$
\begin{aligned}
\boldsymbol{M}= & {\left[\begin{array}{cc}
\boldsymbol{I}_{n}+w_{10^{-1}} \boldsymbol{g g}^{T} \boldsymbol{R}_{1} & -\boldsymbol{\Phi} \\
\boldsymbol{\Phi}^{T} \boldsymbol{R}_{1} & w_{0} \boldsymbol{I}_{n}+w_{20} \boldsymbol{h h}^{T}
\end{array}\right] ; } \\
& 2 n \times 2 n \text { 行列, } \boldsymbol{M}^{-1}=\left[\begin{array}{ll}
\boldsymbol{M}_{11}{ }^{-1} & \boldsymbol{M}_{12}{ }^{-1} \\
\boldsymbol{M}_{21}{ }^{-1} & \boldsymbol{M}_{22}{ }^{-1}
\end{array}\right]
\end{aligned}
$$

ここで, $\boldsymbol{R}_{i}$ は $n \times n$ 対称正定行列, $\boldsymbol{b}_{i}$ は $n$-ベクト ルであり，それぞれ次の差分方程式の解で与えられる.

$$
\begin{aligned}
\boldsymbol{R}_{i}= & w_{2 i} \boldsymbol{h} \boldsymbol{h}^{T}+\Phi^{T} \boldsymbol{R}_{i+1}\left(\boldsymbol{I}_{n}+w_{1 i} \boldsymbol{g} \boldsymbol{g}^{T} \boldsymbol{R}_{i+1}\right)^{-1} \boldsymbol{\Phi} \\
& i=1,2, \cdots, N-1, \quad \boldsymbol{R}_{N}=w_{2 N} \boldsymbol{h} \boldsymbol{h}^{T} \\
\boldsymbol{b}_{i}= & w_{2 i} \boldsymbol{h} z_{i}+\boldsymbol{\Phi}^{T}\left(\boldsymbol{I}_{n}+w_{1 i} \boldsymbol{R}_{i+1} \boldsymbol{g} \boldsymbol{g}^{T}\right)^{-1}\left(\boldsymbol{b}_{i+1}\right. \\
& \left.-\boldsymbol{R}_{i+1} \boldsymbol{g} v_{i}\right), \quad i=1,2, \cdots, N-1, \\
& \boldsymbol{b}_{N}=w_{2 N} \boldsymbol{h} z_{N}
\end{aligned}
$$

証明ほ付録参照.

以上によって $\hat{u}_{i}{ }^{m}, \hat{\boldsymbol{x}}_{i}{ }^{m}$ が求まるとこれらは未知パ ラメータ $\boldsymbol{h}, \boldsymbol{k}$ の関数となるから, (2)の手順は (4)〜 (8) 式の拘束の下で (9) 式の評価関数を最小にする $\boldsymbol{h}, \boldsymbol{k}$ を求めれば良い。

$$
\begin{aligned}
\hat{J}(\boldsymbol{h}, \boldsymbol{k})= & \frac{1}{2}\left[\left\|\hat{\boldsymbol{x}}_{0}{ }^{m}-\overline{\boldsymbol{x}}_{0}{ }^{m}\right\|^{2} w_{0}+\sum_{i=0}^{N-1}\left(v_{i}-\hat{u}_{i}{ }^{m}\right)^{2} w_{1 i}\right. \\
& +\sum_{i=0}^{N}\left(z_{i}-\boldsymbol{h}^{T} \hat{\boldsymbol{x}}_{i}{ }^{m}\right)^{2} w_{2 i}
\end{aligned}
$$

しかし，一般に（9）式は $\boldsymbol{h}, \boldsymbol{k}$ について非線形関数 になり，これを解析的に最小化することは困難である。 また， $N$ が有限な場合（9）式は $\boldsymbol{h}, \boldsymbol{k}$ について一般 そは Unimodal にならないので, 最急傾斜法, 共役 こう配法などの探求法を用いることはできないが，格 子探索とか不規則探索などによる数值計などで最適な $\boldsymbol{h}, \boldsymbol{k}$ の值を求めることができる. 最適な $\boldsymbol{h}, \boldsymbol{k}$ が求ま ると, これに対する $\hat{u}_{i}{ }^{m}, \hat{\boldsymbol{x}}_{i}{ }^{m}$ により過去の入力と状 態の推定值が与觉られる.

\section{3. 重み係数 $\boldsymbol{w}_{1 i}, \boldsymbol{w}_{2 i}$}

前章で，(2) 式の評価関数に沶ける重㕛係数 $w_{0}$, $w_{1 i}, w_{2 i}$ は同定の目的と条件によって与えられると述 ベたが，ここで，これについて検討するとともに（2） 式の評価関数と最尤推定法との関連について考察する.

\section{$3 \cdot 1$ 入力と初期状態に制限がある場合}

従来の実応答誤差を用いた評価関数,

$$
J_{r}=\frac{1}{2} \sum_{i=0}^{N}\left(z_{i}-y_{i}^{m}\right)^{2} w_{2 i}
$$

ただし， $w_{2 i}$ は与えられたとする。

を(1) 式の拘束の下で最小にする同定問題に扣いて, 物理的条件から，測定されたプラントの入力 $v_{i}$ とモ デルの入力 $v_{i}{ }^{m}$ との差に対する制限条件,

$$
\left(u_{i}-u_{i}{ }^{m}\right)^{2} \leq c_{i} \text { ， または } \sum_{i=0}^{N-1}\left(v_{i}-u_{i}{ }^{m}\right)^{2} \leq c
$$

と初期状態に対する制限条件,

$$
\left\|\boldsymbol{x}_{0}{ }^{m}-\overline{\boldsymbol{x}}_{0}{ }^{m}\right\|^{2} \leq d
$$

がつけ加わった場合， $1 / 2 w_{1 i}$ または $1 / 2 w_{1}$ （この場 合 $w_{1 i}=$ 定数 $\left.=w_{1}\right)$ と $1 / 2 w_{0}$ をラグランジュ乗数と 考えると，(1) 式の拘束の下に最小化すべき評価関数 は（2）式のようになる。したがって，この場合の $w_{1 \imath}$ または $w_{1}$ 拈よび $w_{0}$ はそれぞれ (11) 式, (12) 式 を満たすように決めれば良い。

\section{$3 \cdot 2$ プラントの構造と測定誤差の統計的性質が知 られている場合 \\ プラントもモデルと同じく $\left(1^{\prime}\right)$ 式のよらに記述さ} れ, Fig. 1 のように, その入力 $u_{i}$ と出力 $y_{i}$ が測定 雑音 $m_{i}, n_{i}$ に污されてそれぞれ $v_{i}, z_{i}$ として測定さ れるとする。

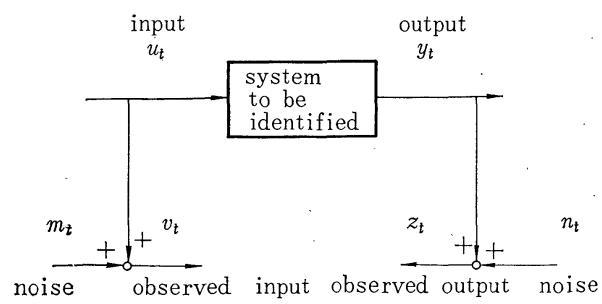

Fig. 1 Linear discrete system

$$
\left.\begin{array}{l}
\boldsymbol{x}_{i+1}=\boldsymbol{\Phi} \boldsymbol{x}_{i}+\boldsymbol{g} u_{i} \\
y_{i}=\boldsymbol{h}^{T} \boldsymbol{x}_{i}
\end{array}\right\}
$$

また，測定雑音 $m_{i} ， n_{i}$ 特よびプラントの初期状態 $\boldsymbol{x}_{0}$ は抎の和のの独立な正規性確率変数であり, その平均 值および共分散が（13）式のように与えられるとする.

$$
\left.\begin{array}{l}
E\left(m_{i}\right)=0, E\left(n_{i}\right)=0 \\
E\left(m_{i} m_{j}\right)=\sigma_{1 i}{ }^{2} \delta_{i j}, E\left(n_{i} n_{j}\right)=\sigma_{2 i}{ }^{2} \delta_{i j}(i, j: 0,1, \cdots) \\
E\left(\boldsymbol{x}_{0}\right)=\overline{\boldsymbol{x}}_{0}, E\left[\left(\boldsymbol{x}_{0}-\overline{\boldsymbol{x}}_{0}\right)\left(\boldsymbol{x}_{0}-\boldsymbol{x}_{0}\right)^{T}\right]=\sigma_{0}{ }^{2} \boldsymbol{I}_{n}
\end{array}\right\}
$$

ただし, $E(\cdot)$ : 空間平均, $\delta_{i j}:$ Kronecker のデルタ. この場合, 入出力の測定データ $v_{i}(i=0,1, \cdots, N-1)$, 
$z_{i}(0,1, \cdots, N)$ が与えられたときの $\boldsymbol{h}, \boldsymbol{k}, \boldsymbol{x}_{0}$ および $u_{i}(i=0,1, \cdots, N-1)$ の最尤推定は (14) 式の条件付 き確率密度関数を $\left(1^{\prime}\right)$ 式の拘束の下で最大にするこ とによって得られる。

$$
\begin{aligned}
& P\left(z_{0}, z_{1}, \cdots, z_{N}, v_{0}, v_{1}, \cdots, v_{N-1}, \boldsymbol{x}_{0} \mid u_{0}, u_{1}, \cdots, u_{N-1}, \theta\right) \\
& =P\left(\boldsymbol{x}_{0} \mid \theta\right) P\left(z_{0}, z_{1}, \cdots, z_{N} \mid u_{0}, u_{1}, \cdots, u_{N-1}, \theta\right) \\
& \quad P\left(v_{0}, v_{1}, \cdots, v_{N-1} \mid u_{0}, u_{1}, \cdots, u_{N-1}, \theta\right) \\
& \text { ただし, } \theta=\left\{\boldsymbol{h}, \boldsymbol{k}, \sigma_{0}^{2}, \sigma_{1 i}{ }^{2}(i=0,1, \cdots, N-1),\right. \\
& \left.\sigma_{2 i}{ }^{2}(i=0,1, \cdots, N)\right\}
\end{aligned}
$$

したがって，一般に maximum likelihood function $L_{N}$ は,

$$
\begin{aligned}
L_{N} & \left(\boldsymbol{x}_{0}, \theta, \mu_{i} ; i=0,1, \cdots, N-1\right) \\
= & \frac{\text { const. }}{\sigma_{0}^{n} \sigma_{2 N} \prod_{i=0}^{N-1} \sigma_{1 i} \cdot \sigma_{2 i}} \exp \left\{-\frac{1}{2}\left[\frac{\left\|\boldsymbol{x}_{0}-\overline{\boldsymbol{x}}_{0}\right\|^{2}}{\sigma_{0}^{2}}\right.\right. \\
& \left.\left.+\sum_{i=0}^{N-1} \frac{\left(v_{i}-u_{i}\right)^{2}}{\sigma_{1 i}{ }^{2}}+\sum_{i=0}^{N} \frac{\left(z_{i}-\boldsymbol{h}^{T} \boldsymbol{x}_{i}\right)^{2}}{\sigma_{2 i}{ }^{2}}\right]\right\}
\end{aligned}
$$

のよらになるから, $\sigma_{0}^{2}, \sigma_{1 i}{ }^{2}(i=0, \cdots, N-1), \sigma_{2 i}{ }^{2}(i=0$, $\cdots, N)$ が既知の場合, $\boldsymbol{h}, \boldsymbol{k}, \boldsymbol{x}_{0}, u_{i}$ の最尤推定は次の 評価関数を(1') 式の拘束の下で最小にすることによ って得られることがわかる.

$$
\begin{aligned}
J_{1}= & \frac{1}{2}\left[\frac{\left\|\boldsymbol{x}_{0}-\overline{\boldsymbol{x}}_{0}\right\|^{2}}{\sigma_{0}^{2}}+\sum_{i=0}^{N-1} \frac{\left(v_{i}-u_{i}\right)^{2}}{\sigma_{1 i}{ }^{2}}\right. \\
& \left.+\sum_{i=0}^{N} \frac{\left(z_{i}-\boldsymbol{h}^{T} \boldsymbol{x}_{i}\right)^{2}}{\sigma_{2 i} i^{2}}\right]
\end{aligned}
$$

（2）式と（16）式を比較してみると（2）式の評価関 数に特ける重み係数 $w_{0}, w_{1 i}, w_{\varepsilon i}$ をそれぞれ $w_{0}=1 / \sigma_{0}{ }^{2}$, $w_{1 i}=1 / \sigma_{1 i}{ }^{2}(i=0, \cdots, N-1), \quad w_{2 i}=1 / \sigma_{2 i}{ }^{2}(i=0, \cdots, N)$ に選ぶと本報告での同定は最尤推定と一致することが わかる。

鞍掛 ${ }^{12)}$ は most probable estimate によって (16) 式と同様な評価関数を用いた同定法を与えているが， most probable estimate ではこのような評価関数が 与えられにくく, また, 初期状態の推定も考慮されて 特らず，Mayne ${ }^{8)}$ の論文との違いがみられない，

$3 \cdot 33 \cdot 2$ と同じ条件で $\sigma_{0}{ }^{2}, \sigma_{1 i}{ }^{2}, \sigma_{2 i}{ }^{2}$ が未知の場合 この場合, 重み係数 $w_{0}, w_{1 i}, w_{2 i}$ を決めることは, $\sigma_{0}^{2}, \sigma_{1 i}{ }^{2}, \sigma_{2 i}{ }^{2}$ を決めることと同じであるから，ここで は, $\sigma_{0}^{2}, \sigma_{1 i}{ }^{2}, \sigma_{\varepsilon i}{ }^{2}$ が未知の場合, これらも未知パラメ 一夕 $\boldsymbol{h}, \boldsymbol{k}$ 之同様に maximum likelihood estimate によって推定することを考える。一般的に，测定雑音 $m_{i}, n_{i}$ は定常であると考光て良いので， $\sigma_{1 i}{ }^{2}=$ 定数 $=\sigma_{1}{ }^{2}, \sigma_{2}{ }^{2}=$ 定数 $=\sigma_{2}{ }^{2}, \nu=\sigma_{1}{ }^{2} / \sigma_{2}{ }^{2}, \nu_{0}=\sigma_{2}{ }^{2} / \sigma_{0}{ }^{2}$ とする と, (15) 式から, $\boldsymbol{h}, \boldsymbol{k}, \nu_{0}, \nu, \sigma_{2}^{2}, \boldsymbol{x}_{0}, u_{i}(i=0,1, \cdots N$ -1) の最尤推定は $\left(1^{\prime}\right)$ 式の拘束の下で (17) 式の評 価関数を最小にすることによって得られることがわか る。

$$
\begin{aligned}
J_{2}= & \frac{1}{2 \sigma_{2}^{2}}\left[\left\|\boldsymbol{x}_{0}-\overline{\boldsymbol{x}}_{0}\right\|^{2} \nu_{0}+\sum_{i=0}^{N-1} \frac{\left(v_{i}-u_{i}\right)^{2}}{\nu}\right. \\
& \left.+\sum_{i=0}^{N}\left(z_{i}-\boldsymbol{h}^{T} \boldsymbol{x}_{i}\right)^{2}\right]+\frac{1}{2} \ln \left[\frac{\nu^{N}}{\nu_{0}{ }^{n}} \sigma_{2}{ }^{4(N+(n+1) / 2)}\right]
\end{aligned}
$$

前章と同様に，まず $\boldsymbol{h}, \boldsymbol{k}, \nu_{0}, \nu, \sigma_{2}{ }^{2}$ が与えられたとし $\tau, u_{i}, \boldsymbol{x}_{i}$ の最適値, $\hat{u}_{i}, \hat{\boldsymbol{x}}_{i}$ を求めると, これらは (18)〜(20) 式のようになる.

$$
\begin{gathered}
\hat{u}_{i}=v_{i}-\nu \boldsymbol{g}^{T}\left(\boldsymbol{R}_{i+1} \hat{\boldsymbol{x}}_{i+1}-\boldsymbol{b}_{i+1}\right), \\
i=0,1, \cdots, N-1
\end{gathered}
$$

$$
\begin{aligned}
& \hat{\boldsymbol{x}}_{i+1}=\left(\boldsymbol{I}_{n}+\nu \boldsymbol{g} \boldsymbol{g}^{T} \boldsymbol{R}_{i+1}\right)^{-1}\left(\boldsymbol{\Phi} \hat{\boldsymbol{x}}_{i}+\nu \boldsymbol{g} \boldsymbol{g} \boldsymbol{b}_{i+1}-\boldsymbol{g} v_{i}\right) \\
& i=0,1, \cdots, N-1 \\
& \hat{\boldsymbol{x}}_{0}=\boldsymbol{M}_{21}{ }^{-1}\left(\nu \boldsymbol{g} \boldsymbol{g}^{T} \boldsymbol{b}_{1}+\boldsymbol{g} v_{0}\right) \\
& +\boldsymbol{M}_{22}{ }^{-1}\left(\boldsymbol{\Phi}^{T} \boldsymbol{b}_{1}+\boldsymbol{h} z_{0}+\nu_{0} \overline{\boldsymbol{x}}_{0}\right)
\end{aligned}
$$

ただし， $\boldsymbol{M}=\left[\begin{array}{cc}\boldsymbol{I}_{n}+\nu \boldsymbol{g} \boldsymbol{g}^{T} \boldsymbol{R}_{1} & -\boldsymbol{\Phi} \\ \boldsymbol{\Phi}^{T} \boldsymbol{R}_{1} & \nu_{0} \boldsymbol{I}_{n}+\boldsymbol{h} \boldsymbol{h}^{T}\end{array}\right]$;

$2 n \times 2 n$ 行列, $\boldsymbol{M}^{-1}=\left[\begin{array}{ll}\boldsymbol{M}_{11}{ }^{-1} & \boldsymbol{M}_{12}{ }^{-1} \\ \boldsymbol{M}_{21}{ }^{-1} & \boldsymbol{M}_{22}{ }^{-1}\end{array}\right]$

ここで, $\boldsymbol{R}_{i}, \boldsymbol{b}_{i}$ は次の差分方程式の解である.

$$
\begin{aligned}
& \boldsymbol{R}_{i}=\boldsymbol{h} \boldsymbol{h}^{T}+\boldsymbol{\Phi}^{T} \boldsymbol{R}_{i+1}\left(\boldsymbol{I}_{n}+\nu \boldsymbol{g} \boldsymbol{g}^{T} \boldsymbol{R}_{i+1}\right)^{-1} \boldsymbol{\Phi}, \\
& \quad \boldsymbol{R}_{N}=\boldsymbol{h} \boldsymbol{h}^{T} \\
& \boldsymbol{b}_{i}=\boldsymbol{h} z_{i}+\boldsymbol{\Phi}^{T}\left(\boldsymbol{I}_{n}+\nu \boldsymbol{R}_{i+1} \boldsymbol{g} \boldsymbol{g}^{T}\right)^{-1}\left(\boldsymbol{b}_{i+1}-\boldsymbol{R}_{i+1} \boldsymbol{g} v_{i}\right), \\
& \quad \boldsymbol{b}_{N}=\boldsymbol{h} z_{N}
\end{aligned}
$$

次に, $\boldsymbol{h}, \boldsymbol{k}, \nu_{0}, \nu_{2}{ }^{2}$ の最適推定は, (18)〜 (22) 式の拘 束の下で (23) 式の評価関数を最小にすることによっ て得られる。

$$
\begin{aligned}
\hat{J}_{2}\left(\boldsymbol{h}, \boldsymbol{k}, \nu, \nu_{0}, \sigma_{2}{ }^{2}\right)= & \frac{1}{\sigma_{2}{ }^{2}} F\left(\boldsymbol{h}, \boldsymbol{k}, \nu, \nu_{0}\right) \\
& +\frac{1}{2} \ln \left[\frac{\nu^{N}}{\nu_{0}{ }^{n}} \sigma_{2}^{4(N+(n+1) / 2)}\right]
\end{aligned}
$$

ただし

$$
\begin{aligned}
F\left(\boldsymbol{h}, \boldsymbol{k}, \nu, \nu_{0}\right)= & \frac{1}{2}\left[\left\|\hat{\boldsymbol{x}}_{0}-\overline{\boldsymbol{x}}_{0}\right\|^{2} \nu_{0}+\sum_{i=0}^{N-1} \frac{\left(v_{i}-\hat{u}_{i}\right)^{2}}{\nu}\right. \\
& \left.+\sum_{i=0}^{N}\left(z_{i}-\boldsymbol{h}^{T} \hat{\boldsymbol{x}}_{i}\right)^{2}\right]
\end{aligned}
$$

$\partial \hat{J}_{2} / \partial \sigma_{2}^{2}=0$ から, $\sigma_{2}^{2}$ の最適值 $\hat{\sigma}_{2}^{2}$ は,

$$
\hat{\sigma}_{2}^{2}=\frac{F}{N+\frac{n+1}{.2}}
$$

となるからこれを（23）式に代入すると，

$$
\begin{aligned}
& \hat{J}_{2}{ }^{\prime}\left(\boldsymbol{h}, \boldsymbol{k}, \nu, \nu_{0}\right)=N+\frac{n+1}{2} \\
& +\frac{N}{2} \ln \left[\frac{\nu}{\nu_{0}^{n / N}}\left(\frac{F}{N+\frac{n+1}{2}}\right)^{2+(n+1) / N}\right]
\end{aligned}
$$

を得る。したがって，最適パラメータ $\hat{\boldsymbol{h}}, \hat{\boldsymbol{k}}, \hat{\nu}_{0}, \hat{\nu}$ は，

$$
J_{0}=\frac{\nu}{\nu_{0}^{n / N}} F\left(\boldsymbol{h}, \boldsymbol{k}, \boldsymbol{\nu}, \nu_{0}\right)^{2+(n+1) / N}
$$


を最小にすることによって得られ， $\sigma_{0}, \sigma_{1}{ }^{2}, \sigma_{2}{ }^{2}$ の最適 推定值 $\hat{\sigma}_{0}, \hat{\sigma}_{1}, \hat{\sigma}_{2}$ は (27) 式で与えられる.

$$
\left.\begin{array}{l}
\hat{\sigma}_{2}^{2}=\frac{1}{N+\frac{n+1}{2}} F\left(\hat{\boldsymbol{h}}, \hat{\boldsymbol{k}}, \hat{\nu}, \hat{\nu}_{0}\right) \\
\hat{\sigma}_{1}^{2}=\hat{\nu} \hat{\sigma}_{2}^{2} \\
\hat{\sigma}_{0}^{2}=\hat{\sigma}_{2}^{2} / \hat{\nu}_{0}
\end{array}\right\}
$$

特に, プラントの初期状態 $x_{0}$ が完全に既知の場合は, $\hat{\boldsymbol{h}}, \hat{\boldsymbol{k}}, \hat{\nu}$ は，(18)，(19)，（21），(22）式の拘束の下で $\left(26^{\prime}\right)$ 式を最小にすることによって得られ， $\hat{\sigma}_{1}^{2}, \hat{\sigma}_{2}{ }^{2}$ は (27') から求められる.

$$
J_{0}{ }^{\prime}=\nu F^{\prime}(\boldsymbol{h}, \boldsymbol{k}, \nu)^{2+1 / N}
$$

ただし，

$$
\left.\begin{array}{rl}
F^{\prime}(\boldsymbol{h}, \boldsymbol{k}, \nu) & \left.=\frac{1}{2}\left[\sum_{i=0}^{N-1} \frac{\left(v_{i}-\hat{u}_{i}\right)^{2}}{\nu}+\sum_{i=0}^{N}\left(z_{i}-\boldsymbol{h}^{T} \hat{\boldsymbol{x}}_{i}\right)\right)^{2}\right] \\
\hat{\sigma}_{2}{ }^{2} & =\frac{1}{N+\frac{1}{2}} F^{\prime}(\hat{\boldsymbol{h}}, \hat{\boldsymbol{k}}, \hat{\nu}) \\
\hat{\sigma}_{1}{ }^{2} & =\hat{\nu} \hat{\sigma}_{2}{ }^{2}
\end{array}\right\} \quad\left(27^{\prime}\right)
$$

以上によって得られた推定值 $\hat{u}_{i}(i=0, \cdots, N-1)$, $\boldsymbol{x}_{i}(i=0, \cdots, N), \hat{\sigma}_{1}{ }^{2}, \hat{\sigma}_{2}$ は, 最尤推定の漸近的性質

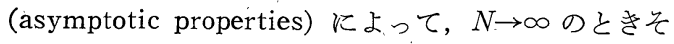
れぞれ真の值に収束することが知られているが(13),14),
観測データが少ない場合には， $\hat{\sigma}_{1}{ }^{2}, \hat{\sigma}_{2}{ }^{2}$ はそれぞれ $E$ $\left(u_{i}-\hat{u}_{i}\right)^{2}, E\left(y_{i}-h^{T} \hat{x}_{i}\right)^{2}$ と同じ程度の推定誤差を伴 うので，本方法により測定雑音の分数を推定するとき はかなり長時間の観測データを用いなければならない。 しかし，本報告は測定雑音の統計的性質が未知な場合 でもパラメータ $\boldsymbol{h}, \boldsymbol{k}$ を有効に推定することを目的と して扣り，これらの推定值 $\hat{\boldsymbol{h}}, \hat{\boldsymbol{k}}$ は $\hat{\sigma}_{1}{ }^{2}, \hat{\sigma}_{2}{ }^{2}$ または重 み係数 $w_{1}, w_{2}$ の值にそれ汪ど影響されないこっを次 章の例題で示す.

以上述べられたほかに，プラント拈よび測定誤差に 関するなえらかの情報も与えられていない場合，2.で 述べた本同定法は，n次元線形離散系のモデルの中か. ら入出力の意味でもっともプラントに近いモデルを求 めることになるが，この場合の重み係数は同定の目的: によって適当に定めればよい。

\section{4. 例 題}

【例 1】(28) 式のように記述される 1 次系のプ ラントに対して $\left(28^{\prime}\right)$ 式のような 1 次係モデルを考え る.

$$
\left.\begin{array}{l}
x_{i+1}=-0.5 x_{i}+u_{i}, x_{0}=0 \text { (既知) } \\
y_{i}=x_{i}
\end{array}\right\}
$$

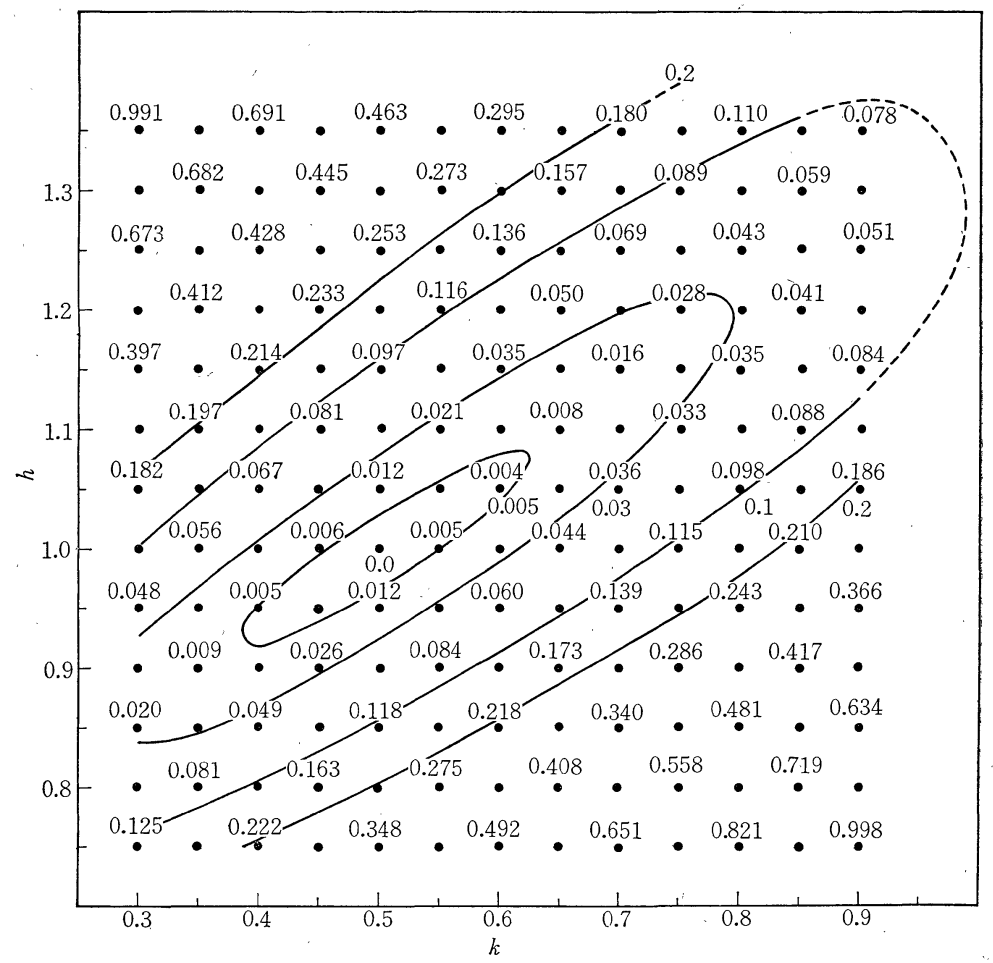

Fig. 2 Level surface of criterion function (eq. 9) for first order system (noises: $0, w_{1 i}$ $=w_{2 i}=1, u_{i}=1, \quad N=15$ ) 


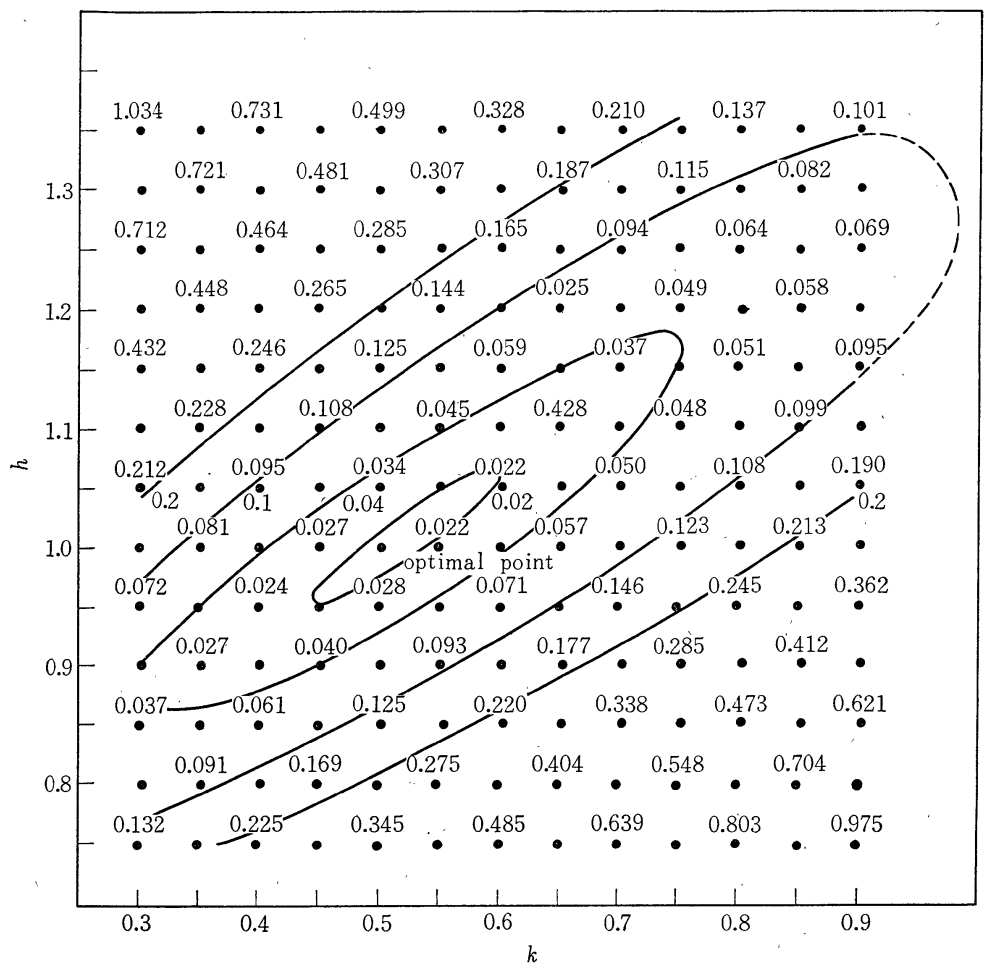

Fig. 3 Level surface of criterion function (eq. 9) for first order system $\left(\sigma_{1}^{2}=0.25 \times 10^{-2}\right.$, $\left.\sigma_{2}^{2}=0.4 \times 10^{-3}, w_{1 i}=w_{2 i}=1, u_{i}=1, N=15\right)$

$$
\left.\begin{array}{l}
x_{i+1}{ }^{m}=-k x_{i}{ }^{m}+u_{i}{ }^{m}, x_{0}{ }^{m}=0 \\
y_{1}{ }^{m}=h x_{i}{ }^{m}
\end{array}\right\}
$$

このプラントの入力 $u_{i}=1(i=0, \cdots, N-1)$ 拉よび出 力 $y_{i}(i=1, \cdots, N)$ が雑音なしで測定されたとする $\left(n_{i}=0, n_{i}=0\right), N=15$ の場合, 重み係数 $w_{1 i}=w_{2 i}=1$ としたときの評価関数 $\left(9^{\prime}\right)$ 式,

$$
\begin{aligned}
\hat{J}^{\prime}(h, k)= & \sum_{i=0}^{N-1}\left(v_{i}-\hat{u}_{i}^{m}\right)^{2} w_{1 i} \\
& +\sum_{i=1}^{N}\left(z_{i}-h \hat{x}_{i}^{m}\right)^{2} w_{2 i}
\end{aligned}
$$

の值をいろいろの $h, k$ の值に対して計算した結果を Fig. 2 に示す. また, 測定誤差 $m_{i}, n_{i}$ が平均値零の 正規乱数でその分散がそれぞれ， $\sigma_{1}{ }^{2}=0.25 \times 10^{-2}$, $\sigma_{2}^{2}=0.4 \times 10^{-3}$ の場合, 同様にして行なった計算結 果を Fig. 3 に示す. このとき， $\hat{h}=1.005, \hat{k}=0.515$ (真の值 ; $h=1, k=0.5)$ となり $\left(9^{\prime}\right)$ 式の最小点が ややずれているのは，短時間データの使用によるもの と考学られる $(N=15)$.

【例 2】例 1 と同じプラントに沶いて, $\sigma_{1}^{2}=0.1$ $\times 10^{-1}, \sigma_{2}^{2}=0.25 \times 10^{-2}(\nu=4)$, 入力 $u_{i}: M$ 系列, $N$ $=30$ の場合, $h, k, \sigma_{2}{ }^{2}, \nu$ を未知として, 3.3 の方法に 従って格子探索を用いてこれらの推定を行なった結果， $\hat{h}=1.021, \quad \hat{k}=0.511, \quad \hat{\sigma}_{1}^{2}=0.446 \times 10^{-2}, \quad \hat{\sigma}_{2}^{2}=0.178$

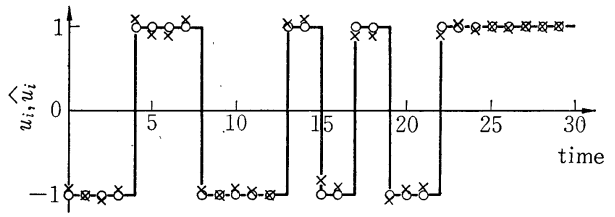

(a). comparison of $u_{i}$ and $\hat{u}_{i}$

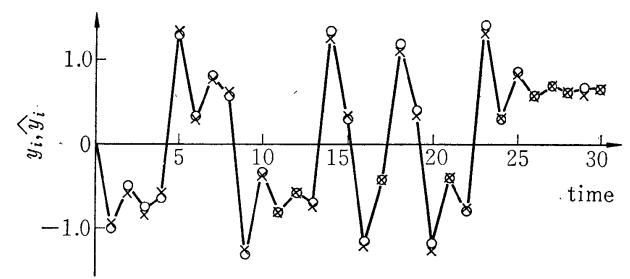

(b) comparison of $y_{i}$ and $\hat{y}_{i}$

$N=30, \hat{h}=1.021, \hat{k}=0.511, \hat{\nu}=250$ ㅇ: true value $\times \times$ : estimated value

Fig. 4 Computations of $\hat{u}_{i}$ and $\hat{y}_{i}$ for first order system when $\sigma_{1}^{2}=0.1 \times 10^{-1}, \sigma_{2}^{2}=0.25 \times 10^{-2}$, and input $: M$-sequence

$\times 10^{-4}, \hat{\nu}=250$ となった. この值に対する $\hat{u}_{i}$ と $u_{i}$ および $\hat{y}_{i}$ と $y_{i}$ との比較をそれぞれ Fig. 4 の (a) (b)に示す. 測定雑音の分散の推定值が真の值から大 きくずれているのは，ここで得られた $\hat{\sigma}_{1}{ }^{2}, \hat{\sigma}_{2}{ }^{2}$ がそれ ぞれ $v_{i}$ 一 $\hat{u}_{i}$ 打よび $z_{i}-h \hat{x}_{i}$ の分散に相当すること, 
短時間データの使用, また $m_{i}$ そ $n_{i}$ が 完全に独立でなく和互いに相関をもつこ となどにより起因するもので，測定デー 夕の個数 $N$ が大きくなり $\hat{x}_{i} \rightarrow x_{i}, \hat{u}_{i} \rightarrow u_{i}$ になるにつれ， $\hat{\sigma}_{1}{ }^{2}, \hat{\sigma}_{2}{ }^{2}$ も文れぞれ $\sigma_{1}{ }^{2}$, $\sigma_{2}{ }^{2}$ に近づくもの之考学られる. 次に

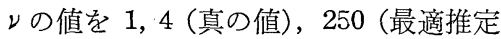
值）之与えて和いて，和の扔のの場合 $h, k$ の推定を行ならと,

$\nu=1$ の場合, $\hat{h}=0.98, \hat{k}=0.49$

$\nu=4 \quad$ " $\quad \hat{h}=0.99, \hat{k}=0.50$

$\nu=250 "$, $\hat{h}=1.02, \hat{k}=0.51$

となった. これよりンの值が $\hat{h}, \hat{k}$ の值 飞それ汪ど大きく影響しないことがわ かる．また， $\nu=1$ および 4 の場合の $\hat{u}_{i}$, $\hat{y}_{i}$ の值も Fig. 4 の $\nu=250$ の場合と㐫 まり相違がなかった。

【例 3】（29)式のように表わせる2 次系プラントに対して, 同じく $\left(29^{\prime}\right)$ 式 の2次系モデルを考光る.

$$
\left.\begin{array}{c}
\boldsymbol{x}_{i+1}=\left[\begin{array}{cc}
0 & 1 \\
-0.5 & 0.85
\end{array}\right] \boldsymbol{x}_{i}+\left[\begin{array}{l}
0 \\
1
\end{array}\right] u_{i}, \\
\boldsymbol{x}_{0}=\left[\begin{array}{l}
0 \\
0
\end{array}\right] \\
y_{i}=[-0.25,0.75]^{T} \boldsymbol{x}_{i}
\end{array}\right\}
$$

$$
\begin{aligned}
& \boldsymbol{x}_{i+1}{ }^{m}= {\left[\begin{array}{cc}
0 & 1 \\
-k_{1} & -k_{2}
\end{array}\right]^{\boldsymbol{x}_{i}{ }^{m}} } \\
&+\left[\begin{array}{l}
0 \\
1
\end{array}\right] u_{i}{ }^{m}, \boldsymbol{x}_{0}{ }^{m}=\left[\begin{array}{l}
0 \\
0
\end{array}\right] \\
& y_{i}^{m}=\left[h_{1}, h_{2}\right] \boldsymbol{x}_{i}{ }^{m}
\end{aligned}
$$

$$
\sigma_{1}^{2}=\sigma_{2}^{2}=0.25 \times 10^{-2}, u_{i}=1, \quad N=30 \text { の }
$$

場合， $h_{1}, k_{1}, x_{0}$ を既知として, $w_{1 i}=w_{2 i}$

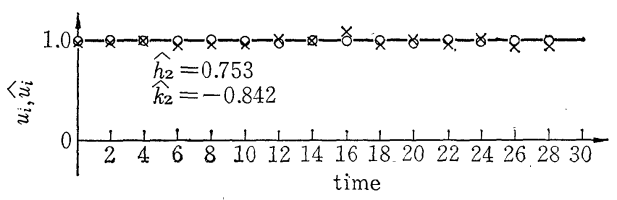

(a) comparison of $u_{i}$ and $\hat{u}_{i}$

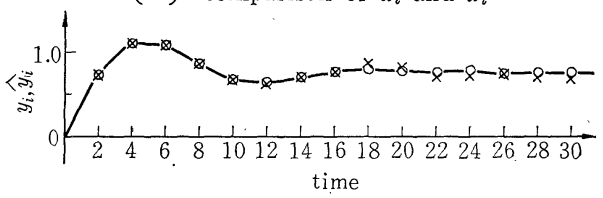

(b) comparison of $y_{i}$ and $\hat{y}_{i}$ $\sigma_{1}^{2}=\sigma_{2}^{2}=0.25 \times 10^{-2}, w_{1 i}=w_{2 i}=1, \quad N=30, \circ$ - : true value, $\times \times$ : estimated value

Fig. 5 Computations of $\hat{u}_{i}$ and $\hat{y}_{i}$ for second order system when $h_{1}, k_{1}, x_{0}$ are known

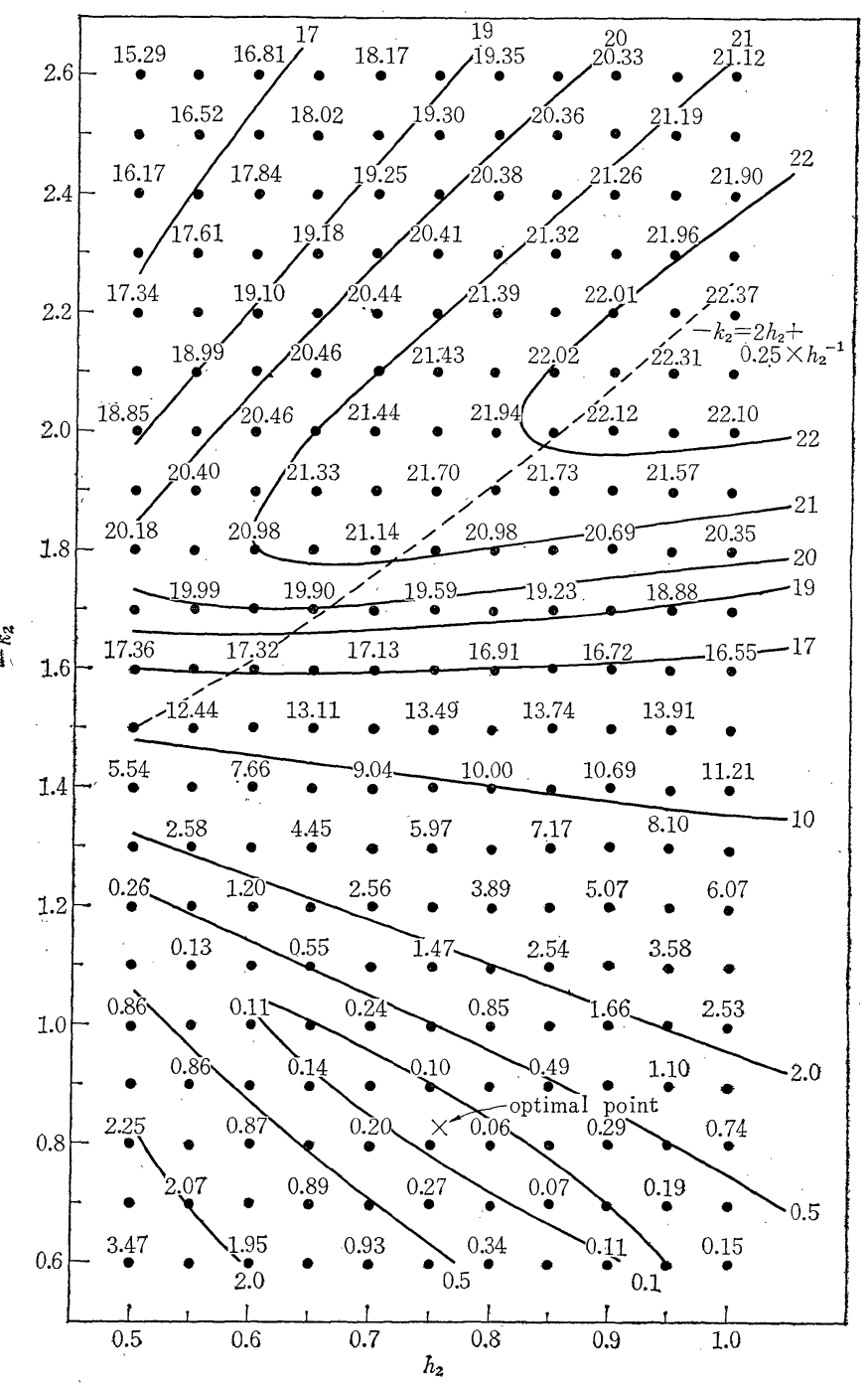

$N=30, \quad \sigma_{1}^{2}=\sigma_{2}^{2}=0.25 \times 10^{-2}, \quad \dddot{h}_{2}=0.753, \quad \hat{k}_{2}=0.842, \quad w_{1 i}=w_{2 i}=1$

Fig. 6 Level surface of criterion function for second order system when $h_{1}, x_{0}$ are known

$=1$ にしたときの $h_{2}, k_{2}$ のいるいろの值に対する評価 関数 $\left(9^{\prime}\right)$ 式の值を Fig. 6 に示す. この場合, $\hat{h}_{2}=$ $0.753, \hat{k}_{2}=0.842$ となった，この值汶する $\hat{u}_{i}\left(\hat{u}_{i}{ }^{m}\right)$

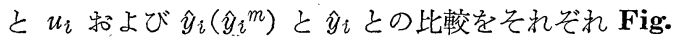
5 の (a), (b) 飞示す. $h_{1}=-0.25, k_{1}=0.5$ のとき, $k_{2}$ と $h_{2}$ が,

$$
k_{2}=-\left(2 h_{2}+\frac{1}{4 h_{2}}\right)
$$

を満足する場合，すなわち Fig. 6 の点線で表わせる 曲線上に物いては，モデルになる $\left(29^{\prime}\right)$ 式の系が可観 测とならず，1次系と等価になるため，評価関数の值 が大きくなるが，これが雑音特よび短時間データの使 用によりずれて，Fig. 6 の上半部の山脈として表われ 
たものと考えられる

5. 結

以上，本報告では有限次元線形モデルを用いてプラ ントを同定するとき，雑音に污されたプラントの入力 信号と出力信号の測定データしか利用できない場合に 対して有効な評価関数を提案した. この評価関数は, プラントの構造と入力信号, 出力信号の測定誤差の統 計的性質が既知の場合, 最尤推定によって得られる評 価関数と一致することが示された. また，与えられた 評価関数の下での測定区間の入力括よび状態の推定を 与觉るアルゴリズムは最適追従制御問題の解を用いて 求められることを示した，乙かし，有限区間のデータ を用いる場合評価関数が パラメータに関し一般には unimodel になる保証がないため，具体的なパラメー タ同定アルゴリズムは与兄られなかった。また，単時 間の観測データを用いる場合，分散の推定値は真值と 大きくずれているにもかかわらず，パラメータの推定 值拉よび入力と出力の推定值は良い精度のむのが兄ら

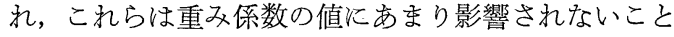
を例題によって示した.

今後，短い測定区間でる評価関数が unimodel にな るような入力信号の設計法拈よび，ここで提案した評 価関数を最小化する具体的探索アレゴリズムなどに対 する研究がなされねばならない。

終わりに, いろいろ御検討, 御助言くださった査読 委員のかたがたに感謝致します。

\section{参考文 献}

1) A. V. Balakrishnan, V. Peterka : Identification in
Automatic Control System, Survey Paper, 4 th Congress of IFAC (1969)

2) P.M. Lion : Rapid Identification of Linear and Nonlinear System, JACC, 605/614 (1966)

3) D. J.Sacrison : The Use of Stochastic Approximation, to Solve System Identification Problem, IEEE, Trans. on AC, AC-12, 513/567 (1967)

4) K. Furuta, J-G, Paquet: On the Identification of Time-Invariant Discrete Process, IEEE, Trans. on AC, AC-15, 153/155 (1970)

5) H. Cox : On the Estimation of State Variables and Parameters for Noisy Dynamic System, IEEE, Trans. on AC, AC-9 5/12 (1964)

6) K. J. Åström (D. Bahlin) : Numerical Identification. of Linear Dynamic Systems from Normal Operating Records, IFAC Symp. on the Theory of Self Adaptive Control System, 97/111, Teddington. (1965)

7) R. L. Kashap : Maximum Likelyhood Identification. of Stochastic Linear Systems, IEEE, Trans. on AC, AC-15-1, 25/34 (1970-Feb.)

8) D. Q. Mayne : Parameter Identification, Automatica, 3, 245/255 (1966)

9) R.E.Kalman : Mathmatical Description of Linear Dynamical Systems, SIAM Control, 1-2, 152/191 (1963)

10) A.E. Bryson \& Y.C. Ho : Applied Optimal Control; Blaisdell (1969)

11）古田 ・河：確定入力を用いる荷重関数の最適同定法, 計測自動制御学会論文集，7-6 572/580 (1971)

12）鞍掛：未知パラメータを含む線形系の推定，計測自動 制御学会論文集，6-6，531/536 (1970)

13) K. J. Áström: On the Achievable Accuracy in Identification Problem, IFAC Symposium on Identification, No. 1.8 (1967)

14) A. Wald : Asymptotic Properties of the Maximum Likelyhood Estimate of an Unknown Parameter of a Discrete Stochastic Process, Ann. Math. Statist., 19, 40/48 (1948)

（1）式の拘束の下で，(A·1）式の評価関数を最小にする問題は，入出力追従最適制御問題である.

$$
J_{\dot{D}}=\frac{1}{2}\left[\left\|\boldsymbol{x}_{0}{ }^{m}-\overline{\boldsymbol{x}}_{0}{ }^{m}\right\|^{2} w_{0}+\sum_{i=0}^{N-1}\left(v_{i}-u_{i}{ }^{m}\right)^{2} w_{1 i}+\sum_{i=0}^{N}\left(z_{i}-\boldsymbol{h}^{T} \boldsymbol{x}_{i}{ }^{m}\right)^{2} w_{2 i}\right.
$$

$J_{D}^{*}$ を,

$$
\begin{gathered}
J_{D}{ }^{*}=J_{D}+\sum_{i=0}^{N-1} \boldsymbol{p}_{i+1}{ }^{T}\left(\boldsymbol{\Phi} \boldsymbol{x}_{i}{ }^{m}+\boldsymbol{g} u_{i}{ }^{m}-\boldsymbol{x}_{i+1}{ }^{m}\right) \\
\text { ただし, } \boldsymbol{p}_{i}(i=0, \cdots, N-1): n \text { 次元ラグランジュ乗数ベクトル }
\end{gathered}
$$

とすると, $J_{D}^{*}$ は，

$$
J_{D}{ }^{*}=\frac{1}{2}\left\|\boldsymbol{x}_{0}{ }^{m}-\overline{\boldsymbol{x}}_{0}{ }^{m}\right\|^{2} w_{0}+H_{0}+\frac{1}{2}\left(z_{N}-\boldsymbol{h}^{T} \boldsymbol{x}_{N}{ }^{m}\right) w_{2 N}-\boldsymbol{p}_{N}{ }^{T} \boldsymbol{x}_{N}{ }^{m}+\sum_{i=1}^{N-1}\left(H_{i}-\boldsymbol{p}_{i}{ }^{T} \boldsymbol{x}_{i}{ }^{m}\right)
$$

ただし， 


$$
\begin{aligned}
H_{i}= & \frac{1}{2}\left[\left(v_{i}-u_{i}{ }^{m}\right)^{2} w_{1 i}+\left(z_{i}-\boldsymbol{h}^{T} \boldsymbol{x}_{i}^{m}\right)^{2} w_{2 i}\right]+\boldsymbol{p}_{i+1}{ }^{T}\left(\boldsymbol{\Phi} \boldsymbol{x}_{i}^{m}+\boldsymbol{g} u_{i}^{m}\right) \\
& \text { (Hamiltonian) } \quad i=0, \cdots, N-1
\end{aligned}
$$

となり， $u_{i}^{m}$ と $\boldsymbol{x}_{i}^{m}$ の最適值をとれぞれ $\hat{u}_{i}^{m}, \hat{\boldsymbol{x}}_{i}^{m}$ とすると，(A.5)〜 (A.7) 式を得る.

$$
\begin{aligned}
\boldsymbol{p}_{i} & =-\left(z_{i}-\boldsymbol{h}^{T} \hat{\boldsymbol{x}}_{i}{ }^{m}\right) \boldsymbol{h} w_{2 i}+\boldsymbol{\Phi}^{T} \boldsymbol{p}_{i+1} \quad(i=1, \cdots, N-1) \\
\hat{u}_{i}{ }^{m} & =v_{i}-w_{1 i}{ }^{-1} \boldsymbol{g}^{T} \boldsymbol{p}_{i+1} \quad(i=0, \cdots, N-1) \\
\hat{\boldsymbol{x}}_{i+1}{ }^{m} & =\boldsymbol{\Phi}_{\boldsymbol{\boldsymbol { x }}_{i}}{ }^{m}+\boldsymbol{g} v_{i}-w_{1 i}{ }^{-1} \boldsymbol{g} \boldsymbol{g}^{\boldsymbol{T}} \boldsymbol{p}_{i+1} \quad(i=0, \cdots, N-1) .
\end{aligned}
$$

また，境界条件は，

$$
\begin{aligned}
& \boldsymbol{\Phi}^{T} \boldsymbol{p}_{1}+\left(w_{0} \boldsymbol{I}_{n}+w_{20} \boldsymbol{h} \boldsymbol{h}^{T}\right) \hat{\boldsymbol{x}}_{0}{ }^{m}=w_{20} \boldsymbol{z}_{0} \boldsymbol{h}+w_{0} \overline{\boldsymbol{x}}_{0}{ }^{m} \\
& \boldsymbol{p}_{N}=-w_{2 N}\left(z_{N}-\boldsymbol{h}^{T} \hat{\boldsymbol{x}}_{N}{ }^{m}\right) \boldsymbol{h} \\
& \text { ただし, } I_{n}: n \text { 次の単位行列 }
\end{aligned}
$$

となる. 次に, $\boldsymbol{R}_{i}$ を 次の対称正定行列， $\boldsymbol{b}_{i}$ を妾べクルとして，

$$
\boldsymbol{p}_{i}=\boldsymbol{R}_{i} \hat{\boldsymbol{x}}_{i}{ }^{m}-\boldsymbol{b}_{i} \quad(i=1, \cdots, N)
$$

と叔き，(A.5)，(A.7)，(A.10) 式から $\boldsymbol{P}_{i+1}, \hat{\boldsymbol{x}}_{i+1}{ }^{m}, \boldsymbol{P}_{i}$ を消去して得られる $\hat{\boldsymbol{x}}_{i}^{m}$ てついての恒等式から，

$$
\begin{aligned}
\boldsymbol{R}_{i} & =w_{2 i} \boldsymbol{h} \boldsymbol{h}^{T}+\Phi^{T} \boldsymbol{R}_{i+1}\left(\boldsymbol{I}_{n}+w_{1 i}{ }^{-1} \boldsymbol{g} \boldsymbol{g}^{T} \boldsymbol{R}_{i+1}\right)^{-1} \boldsymbol{\Phi} \\
\boldsymbol{b}_{i} & =w_{2 i} \boldsymbol{h} \boldsymbol{z}_{i}+\boldsymbol{\Phi}^{T}\left(\boldsymbol{I}_{n}+w_{1 i}{ }^{-1} \boldsymbol{R}_{i+1} \boldsymbol{g} \boldsymbol{g}^{T}\right)^{-1}\left(\boldsymbol{b}_{i+1}-\boldsymbol{R}_{i+1} \boldsymbol{g} v_{i}\right) \quad(i=1, \cdots, N)
\end{aligned}
$$

を得る。また，境界条件は（A·9)，(A·10) 式から，

$$
\boldsymbol{R}_{N}=w_{2 i} \boldsymbol{h} \boldsymbol{h}^{T}, \boldsymbol{b}_{N}=w_{2 i} \boldsymbol{h}_{z_{i}}
$$

となる. 次に（A·10）式を（A·6)，(A·7) 式に代入すると $\hat{u}_{i}{ }^{m}, \hat{\boldsymbol{x}}_{i}{ }^{m}$ は，

$$
\begin{aligned}
\hat{u}_{i}{ }^{m} & =v_{i}-w_{1 i}{ }^{-1} \boldsymbol{g}^{T}\left(\boldsymbol{R}_{i+1} \hat{\boldsymbol{x}}_{i+1}{ }^{m}-\boldsymbol{b}_{i+1}\right) \\
\hat{\boldsymbol{x}}_{i+1}{ }^{m} & =\left(\boldsymbol{I}_{n}+w_{1 i}{ }^{-1} \boldsymbol{g} \boldsymbol{g}^{T} \boldsymbol{R}_{i+1}\right)^{-1}\left(\boldsymbol{\Phi} \hat{\boldsymbol{x}}_{i}^{m}+\boldsymbol{g} v_{i}+w_{1 i}{ }^{-1} \boldsymbol{g} \boldsymbol{g}^{T} \boldsymbol{b}_{i+1}\right) \quad(i=0, \cdots, N-1)
\end{aligned}
$$

で与兄らる。. また， $\boldsymbol{p}_{1}=\boldsymbol{R}_{1} \hat{\boldsymbol{x}}_{1}-\boldsymbol{b}_{1}$ を（A·8）式代入して，これと，(5) 式の $i=0$ の場合とから $\hat{\boldsymbol{x}}_{0}^{m}$ を求める 之（6）式のよらになる.

$$
\hat{\boldsymbol{x}}_{0}{ }^{m}=\boldsymbol{M}_{21}{ }^{-1}\left(w_{10}{ }^{-1} \boldsymbol{g} \boldsymbol{g}^{T} \boldsymbol{b}_{1}+\boldsymbol{g} v_{0}\right)+\boldsymbol{M}_{22}{ }^{-1}\left(\boldsymbol{\Phi}^{T} \boldsymbol{b}_{1}+w_{20} \boldsymbol{h} z_{0}+w_{0} \overline{\boldsymbol{x}}_{0}{ }^{m}\right)
$$

ただし, $\boldsymbol{M}=\left[\begin{array}{cc}\boldsymbol{I}_{n}+w_{10^{-1}} \boldsymbol{g} \boldsymbol{g}^{T} \boldsymbol{R}_{1} & -\boldsymbol{\Phi} \\ \boldsymbol{\Phi}^{T} \boldsymbol{R}_{1} & w_{0} \boldsymbol{I}_{n}+w_{20} \boldsymbol{h} \boldsymbol{h}^{T}\end{array}\right]: 2 \boldsymbol{n} \times 2 \boldsymbol{n}$ 行列, $\boldsymbol{M}^{-1}=\left[\begin{array}{cc}\boldsymbol{M}_{11}^{-1} & \boldsymbol{M}_{12}{ }^{-1} \\ \boldsymbol{M}_{21}{ }^{-1} & \boldsymbol{M}_{22}{ }^{-1}\end{array}\right]$ 